\title{
An unusual case: Adult giant pyonephrosis
}

\author{
Ismail Selvi*, Ali Ihsan Arik and M Sinan Basay \\ Department of Urology, Abdurrahman Yurtaslan Ankara Oncology Training and Research Hospital, Ankara, Turkey
}

\begin{abstract}
Pyonephrosis is a disease, causing suppurative destruction of the renal parenchyma. Fever, chills, and flank pain are most commonly seen. Clinical presentation can include a huge spectrum from asymptomatic bacteriuria (15\%) to urinary sepsis, septic shock and death. Palpable abdominal mass, associated with the hydronephrotic kidney can be detected during physical examination. Urinary system infections, obstruction and anatomic variations (such as pelvic kidney, horseshoe kidney) play a role in etiology. We report a 67-year-old woman with giant pyonephrosis accompanied by a huge intraabdominal mass.
\end{abstract}

\section{Introduction}

Pyonephrosis is a life-threatening disease, causing suppurative destruction of the renal parenchyma. Pyonephrosis may present many times with fever, flank pain and hydronephrosis. Upper urinary tract infection and obstruction play roles in the aetiology of pyonephrosis. Septic shock and death can occurunless it is treated urgently [1]. Due to the high risk of sepsis, early recognition and treatment are extraordinary important. The main treatment is total simple nephrectomy, regardless of aetiology. The general rule is emergency drainage to prevent the risk of sepsis when the upper urinary system remains under pressure. But in case of degraded biological state, both percutaneous nephrostomy catheters and retrograde ureteral internal stents are equally effective for drainage [2]. We report a case of giant pyonephrosis because of its rarity and mimicking the other intraabdominal cystic masses.

\section{Case report}

A 67-year-old woman presented with a 9-months history of left flank pain, progressive abdominal distension and recurrent urinary tract infections. His medical history was unremarkable, except vaginal hysterectomy 10 years ago. Physical examination revealed a temperature of $39^{\circ} \mathrm{C}$, respiratory rate of 18 breaths per minute, blood pressure of $110 / 71 \mathrm{mmHg}$, heart rate of 87 beats per minute, oxygen saturation of $96 \%$ on room air, and capillary blood glucose of $120 \mathrm{mg} / \mathrm{dL}$. On inspection, the abdomen was grossly distended. The physical examination revealed a huge mass at left lumbal region. The palpable mass extended until midline, upper margin was at the level of epigastrium and lower limit was the symphisis pubis. Examination of other systems were normal. The serum analysis and complete blood count was performed. Blood urea was $37 \mathrm{mg} / \mathrm{dL}$, creatinine was 0.97 $\mathrm{mg} / \mathrm{dL}$, hemoglobin was $8.6 \mathrm{~g} / \mathrm{dl}$, hematocrit was $26.8 \%$, white blood cell count was $16.4 \times 10^{9} / \mathrm{L}$, platelet count was $448 \times 10^{9} / \mathrm{L}$ and C reactive protein (CRP) was $320 \mathrm{mg} / \mathrm{L}$. Results of urine analysis were compatible with infection, including abundant leukocytes. We did not think a urinary sepsis because vital parameters were not consistent with sepsis. The low hemoglobin levels were evaluated as anemia of chronic disease. We could not find other reasons to explain the anemia.

Ultrasound scan (US) showed a huge left cystic mass and no signs of renal parenchyma were detected. Computed Tomography (CT) revealed a huge lobulated left cystic mass $(230 \times 149 \times 145 \mathrm{~mm})$ with multiple thin septa. Renal parenchyma was quite thin, almost immeasurably. The intestines were shifted to the right side of the abdomen by the renal mass (Figure 1). Clear distinction with multilocular cystic nephroma, cystic malignancies or cystic hydronephrotic changes could not be made in the tomography report. But appearance suggested a giant hydronephrotic non- functioning kidney.

Patient was consulted to infectious diseases specialist. Blood and urine cultures were taken from the patient. Divided into three equal doses a day, intravenously, $1500 \mathrm{mg}$ meropenem was started empirically. 3 units of blood were transfused to the patient. By administration parenteral antibiotic therapy and blood replacement, fever has gone in two days. No growth was seen in any of the preoperative urine and blood cultures. But the patient was continued on $1500 \mathrm{mg}$ meropenem, intravenously for a week. Ten days after application, open simple nephrectomy was performed via flank incision with retroperitoneal approach. Approximately $2500 \mathrm{ml}$ purulent fluid was drained from the pelvicalyceal system. The ureter was normal as far as can be seen. No obstructive pathology, concerning the ureter was determined (Figure 2).

The patient had an uneventful postoperative period. She was continued on $1500 \mathrm{mg}$ meropenem, intravenously for a week and $0.5 \%$ metronidazole $100 \mathrm{ml}, 4$ times a day was added to the treatment. She was discharged on the $7^{\text {th }}$ postoperative day. According to the pathology report, macroscopically, nephrectomy materialwas $19 \times 16$ $\times 14 \mathrm{~cm}$ in size, brown and off-white in color, showing cystic enlarged nodularity. Histologically, the cystic mass revealed severe dilatation of the pelvicalyceal system with findings of chronic pyelonephritis.But as interesting, no growth was seen in cultures taken from the purulent fluid in the kidney. We could not explain the reason of chronic pyelonephritis. Perhaps it regressed with preoperative antibiotic treatment.

*Correspondence to: Ismail Selvi, Abdurrahman Yurtaslan Ankara Oncology Training and Research Hospital, Department of Urology, Ankara, 06200, Turkey, Tel: +903123360909; E-mail: ismselvi33@hotmail.com

Key words: giant pyonephrosis; non-fonctional kidney; simple nephrectomy

Received: July 10, 2018; Accepted: July 26, 2018; Published: July 31, 2018 


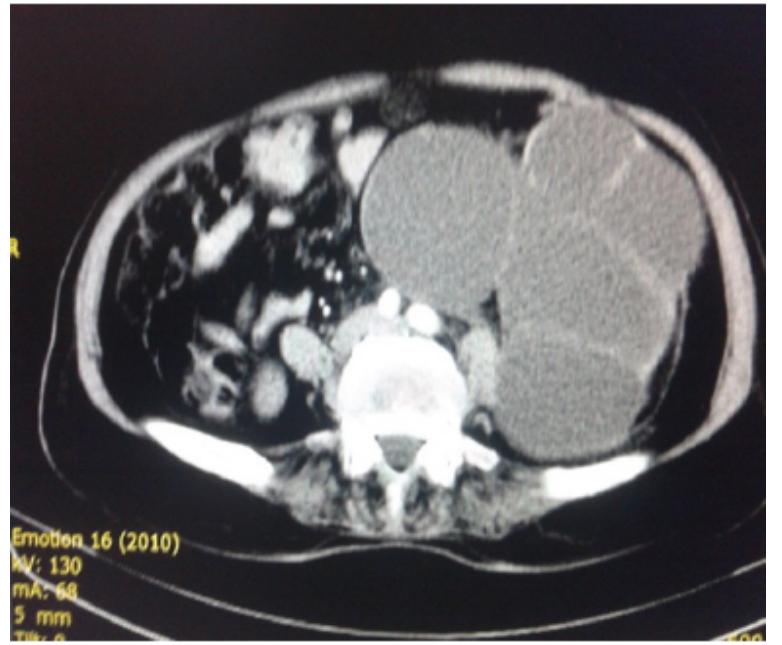

Figure 1. Computed Tomography appearance of the giant hydronephrotic non-functioning kidney. It revealed lobulated left cystic mass $(230 \times 149 \times 145 \mathrm{~mm})$ with no evidence of renal parenchyma

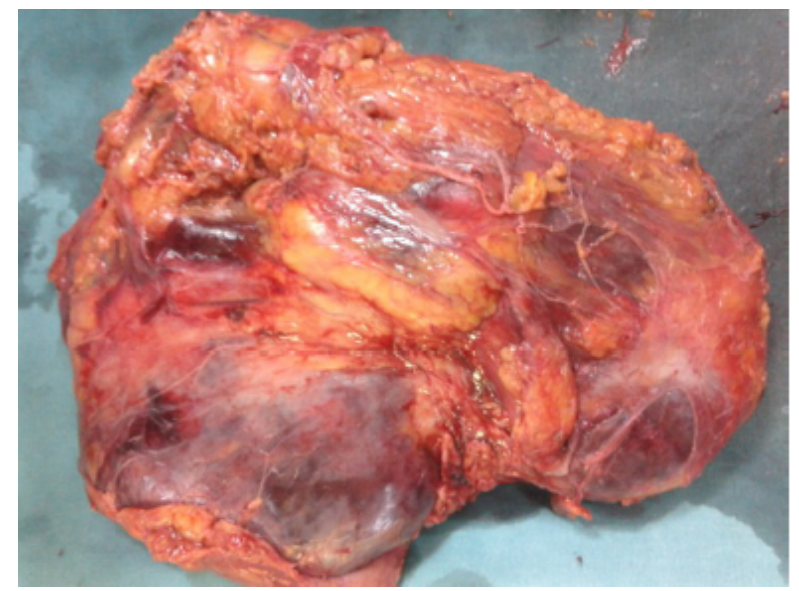

Figure 2. Morphological appearance of nephrectomy specimen. After aspiration of the purulent fluid in the pelvicalyceal system, it was measured $19 \times 16 \times 14 \mathrm{~cm}$ in size

\section{Discussion}

Pathophysiology of pyonephrosis consists of accumulation of purulent exudate in the hydronephrotic collecting system and abscess formation. Pyonephrosis develops on a background of uriner system infections, stones (staghorn in $75 \%$ ) or chronic pyelonephritis, occurred due to obstructions. The common reasons of obstruction can be stones,metastatic tumors (testicular cancer, colon cancer, etc.), yeast balls, pregnancy, and ureteropelvic junction obstruction (UPJO) [3]. Erol et al. [4] reported a giant pyonephrosis with 7 liters in 2014. We did not find any other case with similar size in the literature.

Pyonephrosis can occur in any age groups with urinary obstruction and superimposed infection. In children, urinary obstruction is mostly associated with UPJO. But Simoni et al. [5] presented an extraordinary case of UPJO associated with duplex kidney in a 75-year-old woman. On the other hand, in adults, urinary tract stones are the main cause of urinary obstruction [6]. Intraluminal (such as upper tract urothelial tumours) or extraluminal tumors (such as infiltrative bladder tumours with obstructionof the ureteral orifice, prostatic carcinoma, retroperitoneal lymphadenopathy, retroperitoneal fibrosis) are another important causes of urinary obstruction with high morbidity. As an example of upper tract urothelial tumours, Pello et al. [7] reported a case of urgent nephrectomy because of pyonephrosis and sepsis. Aetiologic factor was sarcomatoid transitional cell carcinoma of the renal pelvis, an infrequent subtype with a poor prognosis In our case, we could not determine any aetiological reason.

Proteus, Escherichia coli, Enterobacter, Klebsiella, Candida and Pseudomonas are the most common organisms in pyonephrosis. It is much more common in females (73\%) than men (27\%) [8]. Pyuria is seen very commonly in pyonephrosis and may sometimes be nonspecific. Bacteriuria, leukocytosis, flank pain and fevermay be absent in $30 \%$ of the cases [1].

The distinction from other septic renal collections (renal abscess, perinephric abscess) is performed using USG, CT or intravenous urography. CT is more effective than USG because renal function, causes of obstruction (stone, retroperitoneal fibrosis, metastatic masses, etc.), and similar abdominal pathologies such as hydronephrosis are better shown in CT [9].

The most terrible complication of pyonephrosis is urosepsis, whose mortalityranges varies between $20 \%$ and $42 \%$ [10]. For management of pyonephrosis, antibiotics have no effects, unless the pus is surgically drained [10].Percutaneous nephrostomy and ureteral catheter insertion are necessary in many cases, especially in acute phase. Wang et al. [11] showed effects of renal pelvic high-pressure perfusion on nephrons in a porcine pyonephrosis model. According to them, percutaneous nephrolithotomy should be performed for renal calculus complicated with severe infection, control of the intraoperative renal pelvic pressure is strictly necessary. Maintaining the pressure below $20 \mathrm{mmHg}$ is also relatively safe. If the contralateral kidney is normal, simple nephrectomy may be performed for unilateral non-functional kidney. If nephrostomy is installed urgently, nephrectomy can be performed with the improvement of the general condition (usually after two weeks) [12,13]. In our case, patient's vital signs (such as fever, flank pain, urine analysis) improved immediately after hydration and parenteral antibiotic therapy. Therefore we did not have need of percutaneous nephrostomy or ureteral catheter initially.

Intraoperative frequent complications are injuries of colon (most commonly on the left),duodenum, inferior vena cava, laceration of inferior pole of the spleen and accidental opening of the pleural or peritoneal cavity $[12,13]$. Postoperative frequent complications are postoperative colic, ileus, profound thrombophlebitis and suppurations of the surgical wound $[12,13]$.

Arvind et al. [14] presented a case of laparoscopic nephrectomy for pyonephrosis during pregnancy in 2011[14]. When pyonephrosis complicates pregnancy, maternal and fetal ill healthshould be considered more carefully thanever. Laparoscopic approach reduces the risk of uterine irritability by decreasing the need for uterine manipulation and is concluded in lower rates of spontaneous abortion and preterm delivery than open surgery. But the major risks of operation are fetal acidosis secondary to $\mathrm{CO} 2$ absorption, alteration in placental perfusion secondary to pneumoperitoneum and possibility of fetal hypotension.

In conclusion, management of pyonephrosis is a vital procedure. Precense of pyonephrosis should be diagnosed early and surgery should be performed immediately after acute phase treatment. Percutaneous nephrostomy can initially be installed for draining the pus from renalcavities, so it reduces the degree of secondary perinephritis.

As an addition to the literature, we think that as in our case, percutaneous drainage may not be initially performed if patient's 
vital signs (such as fever, respiratory rates, blood pressure, heart rates, oxygen saturation, flank pain, urine analysis) improve immediately after hydration and parenteral antibiotic therapy. But simple nephrectomy is the main surgical treatment option if contralateral kidney is healthy. It is also necessary in cases in which the patient has singlefunctional kidney, patient will be on dialysis.

\section{References}

1. Eroglu M, Kandirali E (2007) Akut Pyelonefrit ve pyonefroz. Turkiye Klinikleri J Surg Med Sci 3: 24-28.

2. Yoder IC, Lindfors KK, Pfister RC (1984) Diagnosis and treatment of pyonephrosis. Radiol Clin North Am 22: 407-414. [Crossref]

3. Rabii R, Joual A, Rais H, Fekak H, Moufid K, et al. (2000) [Pyonephrosis: diagnosis and treatment: report of 14 cases]. Ann Urol (Paris) 34: 161-164. [Crossref]

4. Erol A, Coban S, Tekin A3 (2014) A giant case of pyonephrosis resulting from nephrolithiasis. Case Rep Urol 2014: 161640. [Crossref]

5. Simoni F, Vitturi N (2014) Sepsis due to pyonephrosis: an adult with pelvic-ureteric junction obstruction (PUJO) in a duplex kidney. J Ultrasound 18: 301-304. [Crossref]

6. Ramchandani P, Cardella JF, Grassi CJ, Roberts AC, Sacks D,et al. (2003) Quality improvement guidelines for percutaneous nephrostomy. Society of Interventiona Radiology Standards of Practice Committee. J Vasc Interv Radiol 14: 277-281. [Crossref]
7. Fernández-Pello S, Venta V, González I, Gil R, Menéndez CL (2014) Pyonephrosis as a sign of sarcomatoid carcinoma of the renal pelvis. World J Clin Cases 2: 215-218. [Crossref]

8. Pearle MS, Pierce HL, Miller GL, Summa JA, Mutz JM, et al. (1998) Optimal method of urgent decompression of the collecting system for obstruction and infection due to ureteral calculi. J Urol 160: 1260-1264. [Crossref]

9. Fultz PJ, Hampton WR, Totterman SM (1993) Computed tomography of pyonephrosis Abdom Imaging 18: 82-87. [Crossref]

10. Dellinger RP, Carlet JM, Masur H, Gerlach H, Calandra T, et al. (2004) Surviving SepsisCampaign guidelines for management of severe sepsis and septic shock. Crit Care Med 32: 858-873. [Crossref]

11. Wang J, Zhou DQ, He M, Li WG, Pang X, et al. (2013) Effects of renal pelvic highpressure perfusion on nephrons in a porcine pyonephrosis model. Experimental and Therapeutic Medicine 5: 1389-1392. [Crossref]

12. Reinhart K, Meisner M, Brunkhorst FM (2006) Markers for sepsis diagnosis: what is useful? Crit Care Clin 22: 503-519, ix-x. [Crossref]

13. Schaeffer AJ, Schaeffer EM (2007) Infections of the Urinary Tract. Campbell- Walsh Urology ninth edition, Philadelphia: Saunders Elsevier. 223-296.

14. Arvind NK, Singh O, Gupta SS, Sahay S, Ali K, et al. (2011) Laparoscopic Nephrectomy for Pyonephrosis During Pregnancy: Case Report and Review of the Literature. Reviews in Urology 13: 98-103. [Crossref]

Copyright: $(02018$ Selvi I. This is an open-access article distributed under the terms of the Creative Commons Attribution License, which permits unrestricted use, distribution, and reproduction in any medium, provided the original author and source are credited. 